\title{
Smart Urban Parking Solution in Sri Lanka
}

\author{
M.D.S.M. Antany \\ Software Engineer, Department of \\ Information Technology \\ SLIIT Malabe Campus, \\ Malabe
}

\author{
Priyankara A.D.D. \\ Software Engineer, Department of \\ Information Technology \\ SLIIT Malabe Campus, \\ Malabe
}

\author{
M.R.M. Aadil \\ Software Engineer, Department of \\ Information Technology \\ SLIIT Malabe Campus, \\ Malabe
}

\author{
M.G.N.M. Pemadasa \\ Assistant Lecturer (Supervisor), \\ Department of Information \\ Technology SLIIT Malabe Campus, \\ Malabe
}

\author{
L.V. Ferreira \\ Software Engineer, Department of \\ Information Technology SLIIT \\ Malabe Campus, \\ Malabe
}

\begin{abstract}
A sustainable, feasible, and affordable mobile application solution to automate the vehicle parking of a driver with features like finding available parking slots, finding the nearest parking area, and navigating to the available parking slot inside a parking area would be beneficial for the drivers since it would save valuable time and money and for the owners, owners would allow utilizing their limited spaces in urban areas to the maximum efficiency increasing their profit rate and saving environmental pollution by limiting the toxic gases produced to the environment. 'Pay as You Park' Smart Parking Solution empowers its users to use above mentioned advantages with its mobile application for drivers and, a web application for car park owners. The main uniqueness is that instead of traditional hourly charged parking fees, introduced system charges only for the exact time been parked. This system uses on-premises CCTV cameras to identify the availability of parking spaces and it allows anyone with a space to park a vehicle to register with the system and contribute the space to the system while earning extra money once the system validated proper conditions of a parking area. With the current growth of the number of vehicles on Sri Lankan roads, traffic has become a huge problem. 'Pay as You Park' Smart Parking Solution saves the time of the user since it enables the user to skip the time-intensive process of finding the perfect parking slot for their vehicle with the use of a Machine Learning-based algorithm which is used to find the suitable parking slot for a particular vehicle.
\end{abstract}

\section{Keywords}

ToA - Time of Arrival, TDoA-Time Difference of Arrival, SVM - Support Vector Machine, GPS - Global Positioning System

\section{INTRODUCTION}

This paper introduces a fully automated smart parking system to cross over the barrier between parking yards and the drivers. With the growth of smart technology, the concept of a smart city is a rapidly growing area under smart concepts. Smart parking solutions play a vital role in the process of smart cities. Though parking is an essential task there is a lack of smart technology used for parking in Sri Lanka which is the main reason why smart parking is not being used by most users. With the survey conducted authors have identified that the majority of the users find that current parking solutions are there to achieve their needs. Though there are newer technologies available today the present parking system fails to overcome by providing optimal results [1].

'Pay as you Park' is a mobile application for drivers or regular users and a web application for parking yard owners. It will be addressing the most issues faced by the parking yard owners and drivers in the process of parking. Also, functionality to register an available space for the 'Pay as You Park' smart parking system will be introduced, and the registration process will be validated and approved by the system. The majority of the users find that the payment algorithm that is used by existing parking systems is not user-friendly in this solution authors are introducing pay-as-you-park for the registered users were to have to pay only for the time which has parked.

The proposed solution introduces a tracking mechanism to identify the available free spaces of parking yards using CCTV cameras in the parking premises. It will minimize the expense of sensors. And optimal parking yard suggestions for the drivers will be given based on the location, availability, physical characteristic of thevehicle, distance, and the time taken to reach the parking destination. Also, 'Pay as you Park' introduces internal navigation inside the parking yards which will be helpful for the users to easily navigate to their parking slots. For the parking yard owners, an automated validating system to validate or approve the quality of the parking space will be shown in the registration phase. With the introduction of these concepts into the 'Pay as you Park' solution; it would be beneficial for both the party's drivers and the parking yard owners. And also, it would give a positive impact on urban society.

\section{LITERATURE REVIEW}

In the literature review, the authors have focused on the same areas of similar research functionalities and parts. The main purpose of this project is to provide an optimal parking experience for both user and owner that is user-friendly and has the intelligence to assist the users in the process of parking.

Many types of research have been conducted regarding smart parking solutions and integrating sensors [2][3] to parking slots in Sri Lanka. These systems have proved that having a system makes the driver's experience better. Current systems use different technologies to identify the occupancy of parking slots in a parking area. Developers utilize technologies such as 
ultrasonic sensors, Arduino-based systems, magnetometers, Infrared sensors, LDR-Light Dependent Resistors, and microcontroller-based systems. But past researchers have found that these systems have their drawbacks such as not suitability to open-outdoor parking slots, object detection issues such as humans, animals, and other objects are being identified as vehicles, and also these devices consume a higher rate of power consumption. [4][5]

And also, on field visits to some parking areas in Sri Lanka, authors have experienced some events that displayed incorrect sensor details such as displaying the parking slot is free when there was a vehicle inside it. And the team has experienced how much time is being consumed for the parking process since the drivers could not find a parking place inside a parking area in extremely urban areas such as Pettah (Colombo 11) and Colpetty (Colombo 03) due to a lack of system.

These accuracy issues and lack of a proper approach to find a vacant space inside a parking space consume a lot of valuable time of the drivers and it burns a lot of money and, past researchers have found on this field have found that drivers spend $30 \%$ of their driving time to park a vehicle. Also, other research has found that drivers spend an average time of 3.5 minutes to 14 minutes to find a free parking space.

'Pay as You Park' smart parking solution considers these issues and helps to overcome these issues for the consumer by providing a mobile app that enables the user to find out the available parking slots inside a parking area and support the driver to save his or her valuable time and money. To offer an affordable and sustainable solution for every end-user of the system, the 'Pay as You Park' team has done some researchers to find out the best technology to use for finding 'Occupancy in a parking area'. After analyzing the researchers, the team has decided that using CCTV footage for the process would be the ideal option since most of the car parking areas already have employed CCTV - surveillance cameras for security purposes so it would save the cost of additional sensors or microcontrollers as primary devices.

Many researchers have been conducted under the domain of parking. The user's location is determined via a Global Positioning System (GPS) device. If the equipment has a clear line of sight to the satellites, it will work in any weather [6]. It shows that GPS technology has high global availability. According to many researchers GPS is widely used to track the user's location. 'Pay as you Park' solution needs to track the location of users to suggest them optimal parking suggestions and navigate them to yards. Authors have been used many Machine Learning algorithms to find the nearest parking location to the user. TOPSIS (Technique for Order of Preference by Similarity to Ideal Solution) algorithm is a multiple-criteria decision-making algorithm [7]. Here authors have used the TOPSIS algorithm to identify the nearest medical service provider for the users. The Haversine theorem is used to calculate the lengths of two points on the surface of the earth based on latitude and longitude [8]. This research was conducted to find the nearest mosque for a user. The author has used the Haversine theorem to identify which is nearest to the user. The haversine algorithm is widely used and recommended by the authors as it is less cost-effective. In the previous research, the authors discussed many algorithms to identify the availability of free spaces in parking yards. Authors of research [9] have discussed a similar aspect to identify the occupancy of a parking yard at a given time. As parking data is available of seasonality; Seasonal ARIMA is identified as a machine learning algorithm that predicts the occupancy with high accuracy.

There are two Range based schemes. Distance Estimation and Position Estimation are two of them. Research attempts have been made in the literature to reduce the distance error and improve the accuracy of the location. The centroid localization algorithm proposed uses the coordination of beacons to estimate the location of the unidentified mobile positioning using the centroid formula. Still, with this algorithm, the position accuracy is inferior. The algorithm for Weighted Centroid Localization uses weight to estimate location as a factor. The AWBCL algorithm based on the WCL algorithm has improved the accuracy of the spot, but the position error is still high.[10] RSSI-based and ToA/ TDoA-based are distance estimation schemes. Below are the pros and cons of the distance estimation methods. Advantages of RSSI are low complexity, No time sync, and low power. The main disadvantage of RSSI is low accuracy but with the help of Kalman filtering, the accuracy can be increased. ToA's main advantage of ToA/ TDoA is high accuracy but the complexity is higher than RSSI and high power. The main reason to use beacons is beacons are cheaper than the other technologies. The Comparison is shown in Table 1.

Table 1 - Technology Comparison

\begin{tabular}{|l|l|l|l|}
\hline $\begin{array}{l}\text { Indoor } \\
\text { Positioning } \\
\text { Service }\end{array}$ & $\begin{array}{l}\text { Technology } \\
\text { Name }\end{array}$ & $\begin{array}{l}\text { Cost/ } \\
\text { Installatio } \\
\mathrm{n}\end{array}$ & Accuracy \\
\hline Radar & WIFI & $\begin{array}{l}\text { Cheap/ } \\
\text { Complex }\end{array}$ & $2-3 \mathrm{~m}$ \\
\hline Beacon & Bluetooth & $\begin{array}{l}\text { Cheap/Not } \\
\text { Complex }\end{array}$ & $\begin{array}{l}0.5-3 \mathrm{~m} \\
\text { (There are } \\
\text { beacons } \\
\text { that have } \\
100 \mathrm{~m} \\
\text { accuracy) }\end{array}$ \\
\hline $\begin{array}{l}\text { LANDMAR } \\
\text { C }\end{array}$ & RFID & $\begin{array}{l}\text { Cheap/Not } \\
\text { Complex }\end{array}$ \\
\hline
\end{tabular}

There are many parking applications available worldwide in different regions, but no one is providing a space for registering new places (e.g., private car parks, lands, garages, etc.) as parking slots on the map. In urban areas, Car parking is a serious issue in both developed and developing counties. Many cities are suffering from a lack of vehicle parking places as a result of the fast rise in car ownership, with an imbalance between parking availability and demand, which may be regarded as the root cause of metropolitan parking issues [11]. Considering the virtual parking solution, it is impossible to manage private properties to use as parking spaces in public use. So that "Pay as you park" smart parking solution introducing a new feature to overcome the lack of parking facilities in urban areas by standardizing the private places for public use. So, the "pay as you park" team has researched the best technology to identify the standard of the private places for parking vehicles before it registers to the "Pay as you park" system.

In this case, detecting potholes and identifying the surface type and the quality of the parking place is the major task of this system when registration of the lands. Otherwise, drivers may encounter a variety of issues when parking in those new locations, causing them to lose faith in new parking places provided by the solution. Many types of research have 
implemented sensor-based systems [12] and machine learning approaches [13][14] to locate potholes, objects, and surface quality. Different authors have been used many machines learning and image processing approaches for different problems such as soil classification, pothole detection, surface cracks detection, etc.

Regarding related work, a group of researchers present a literature review about recent publications for soil type classification. In this approach, researchers developed a model and image processing-based soil classifier to identify the soil type of the ground. In that model, researchers have used seven classes of soil for classification and the preprocessed images have extracted the features, and the data extracted is used to train the Support Vector Machine (SVM) classifier [14].

In another research, a CNN-based road surface crack detection model was developed which responds to the brightness

changes. In that case, researchers come up with a semantic segmentation model with an autoencoder structure for detecting road surface along with a CNN-based image preprocessing model as a solution to the road surface crack detecting under the different lighting conditions of the images [15].

Another experiment was carried out by a group of researchers to find the best accurate model for monitoring road surfaces and detecting the pothole. In that work, researchers have analyzed and applied different deep learning models: convolutional neural networks $(\mathrm{CNN}), 4$ Long short-term memory network (LSTM) networks, and reservoir computing (RC) models. At the end of the research, researchers have shown the confusion matrices and accuracy for each evaluated model. And researchers proved that in contrast to $\mathrm{CNN}$, the other models failed to classify the rest of the classes, and most potholes were correctly classified by themselves [16].

\section{RESEARCH OBJECTIVES}

The main objective of this research is to introduce a userfriendly automated smart parking solution for both drivers and parking yard owners. And increase the productivity of urban parking in Sri Lanka which will be a solution for most of the environmental and social problems.

\subsection{Tracking Available Parking Spaces}

This system identifies the availability of parking slots of a parking area using CCTV footages of a parking yard. The data of the availability is sent to the system for further processing.

\subsection{Optimal Parking Yard Suggestions}

'Pay as you Park' smart parking system identify and suggest the most optimal parking yard for a vehicle based on the current location, availability of free spaces in parking yards, physical characteristics of vehicles like (height, width, length), and the time taken to reach the parking yard.

\subsection{Internal Navigation}

The introduces smart parking system, 'Pay as you Park' guides users when a user drives into the parking area by showing the free parking slots on a map to drive the vehicles to the available parking slot. Although the indoor navigation is not accurate due to obstacles like walls and ceilings, this system is implemented by using a cheap and small device called a Beacon. Beacons are cheap when consider to other indoor positioning devices and also this gives much more accuracy than the other positioning technologies like Wi-Fi, etc.

\subsection{Parking Yard Surface Validation}

Land suitability evaluation is one of the important procedures before setting that place for car parking. So that people have to waste more time and money for that to take advice from their expertise. When considering the real-world scenario of a car parking solution, registering, and verifying the suitability of new land for a car parking application would not easily process for the system admin in his busy daily routine. And, when considering the world map there are millions of lands and garages are owned by people in different regions. So, once the registration begins after a Parking application deployment and if this process is maintained by a human being, there would be a huge queue for private land and garage registration and verification processes. Therefore, to overcome these problems, machine learning and image processing-based automated method is going to introduce in this paper. Using this method, the parking system can analyze the parking owner's image data of the parking land/garage, which was taken while the registration process, and provide the suitability level of the ground and the necessary suggestion to resubmit images after changes are done by the parking lot owner.

\section{METHODOLOGY}

\subsection{High-Level Architecture}

Overall research contains a unique web-based system that supports both desktops and mobiles. There are mainly three types of users Clients (Drivers), Car Park owners, and Moderators (Admin). The suggested method outlines a stepby-step procedure for bridging the gap between drivers and car park owners. This application will serve as a common platform for all three parties (drivers, car park owners, and moderators), ensuring that the need for parking availability demanded by drivers and car parks is met. The proposed method is the most effective solution for Sri Lankan Road traffic jams and daily parking issues faced by drivers.

\subsection{Tracking Available Parking Spaces}

As discussed earlier in the literature review, the current car parking system now utilizes several sensors and microcontrollers to identify the occupation of parking slots inside a parking area. The 'Pay as You Park' smart parking solution provides a greener and feasible resolution to the current difficulties occurring while finding a parking slot for a driver. This system uses CCTV footage and analyzes the availability of a parking slot inside a parking area. After the literature survey, authors have discovered that there are several algorithms to identify the available parking slots with image processing. CNN, R-CNN(RCNN), Faster RCNN with Region Proposed Networks, and finally Mask R-CNN which was the ideal algorithm to use. CNN or Convolutional Neural Network is a kind of artificial neural network employed in image processing that is optimized to pixel data. CNN are the basic building blocks for the visual task of image segmentation. R-CNN (Regional CNN) is a type of Machine learning model which is used for computer vision tasks and the importance of RCNN is that it is particularly used for object detection. RCNN classifies several picture regions into the suggested class by using bounding boxes across object regions and then deciding on convolutional networks independently on all Regions of Interest (ROI). Mask RCNN is a Convolutional Neural Network that was built on the foundations of Faster RCNN (RCNN improvement) and a Region-based Convolutional Neural Network. Since it is an extension of Faster RCNN, it has an additional mask, the object mask apart from two other outputs in Faster RCNN, a 
class label, and a bounding box offset. Mask R-CNN has considerable characteristics which demonstrate it is the absolute model to use such as easiness to train, high performance, efficiency, and it is easy to generalize to other tasks.[17]

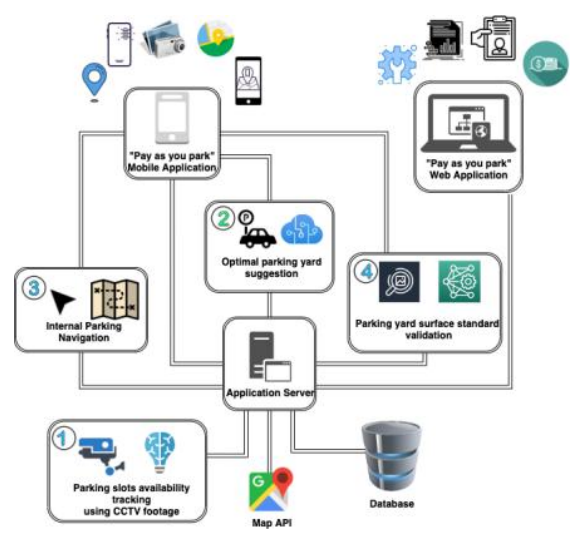

Figure 1 - System Hardware Architecture Diagram

\subsection{Optimal parking yard suggestion}

Most of the parking yards that have been introduced are commonly considering the location and distance to the parking yard. But in 'Pay as you Park' smart parking solutions will be considered on availability, location, distance, and physical characteristics of the vehicle. And also, it is considered that the amount of time taken to reach the destinated parking yard and will make sure there is available free space to park the vehicle. To track the users' current location and navigate them to their destination; GPS and google Maps API services will be used. Since the system is based on mobile application GPS services provided by mobile phones can be used for tracking the location. With the literature survey authors identified that there are a huge number of algorithms available to calculate distance and identify the nearest to a particular user or a destination. Haversine, K Nearest Neighbor (KNN), TOPSIS, and Dijkstra's algorithm are being rapidly used. Considering the accuracy and computing power Haversine algorithm used to identify the nearest parking yard, although assumptions in the algorithm will affect the real distance, it will not affect the results authors expect. Authors have to use a time series forecasting prediction with machine learning to predict the availability of a parking yard at a given time and date.

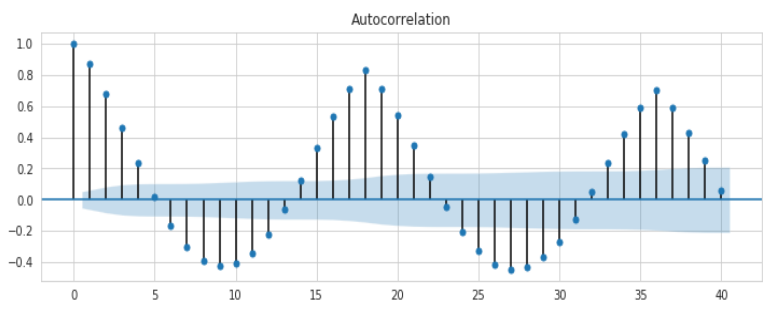

Figure 2 - Correlation in parking data

As shown in figure 2, the parking data possess a seasonality with it, the most accurate forecasting algorithm would be the Seasonal Autoregressive Integrated Moving Average (SARIMA). Using SARIMA the percentage of occupancy of a parking yard at a given date and time would be predicted. Based on the prediction 'Pay as you Park' smart parking solution will suggest the optimal parking yard to park their vehicle based on the above-discussed facts.

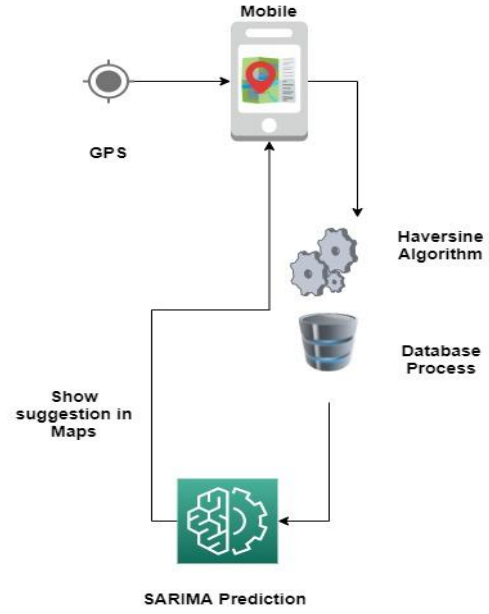

Figure 3 - Optimal Suggestion Process

\subsection{Internal Navigation}

According to the current systems in Sri Lankan drivers are wasting their time when finding the exact free slot inside a parking lot after getting into a parking lot. In this system, after users get into the parking lot, from the mobile application user must select the name of the parking area. After users choose the parking area, and then after the system shows a map of the parking area and the available free slots inside the parking area. Free parking slots are getting from the database details which are handled by objective A and those available parking slots will be visible to the users on the map. Although a user outside can be tracked by using technology like GPS it is hard to track or cannot track a user's position accurately while the user is inside a building or underground due to some obstacles like walls, ceilings, etc. By reviewing many indoor positioning systems, in this method, Beacons are being used. According to the literature review, beacons are cheaper than the other technologies and Beacons are more accurate.[18] To get the users positioning three beacons are used with the help of RSSI values of the beacons and Neural Network Machine Learning Algorithm. In this method getting the distance from mobile to each beacon by using the BLE RSSI values of each beacon and passing it to the trained model. Then after the user's $\mathrm{X}$ and $\mathrm{Y}$ values are being predicted by the model. After getting the $\mathrm{X}$ and $\mathrm{Y}$ values of the users these values are displayed on the map. Then the user can see the user's position and the user can find the way to the free parking slot shown on the map. Assume there are two stories in a particular building and how are users able to identify the floor? Yes, that would be a concern. To avoid a few beacons can be used. Beacons have unique ID's and to identify the floors the IDs of those beacons can be used.[19] The internal Navigation diagram structure is shown in Figure 4.

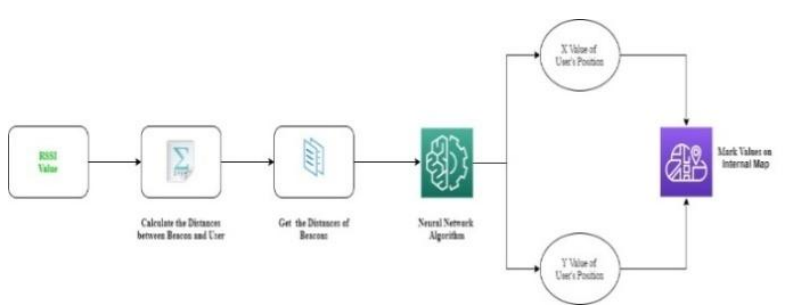

Figure 4 - The structure of user tracking.

\subsection{Parking yard surface validation}

When considering the process of the parking yard registration to the system, it is needed to verify whether the parking place 
surface is at least the minimum standard to park a vehicle in that place. In that case, the suggested solution was to come up with a monthly based automated parking place quality checking method in the system. This methodology works when the parking owner is trying to register his parking yard/garage to the system for renting and the end of the month quality checking of the parking yard to continue the business. So, to accomplish this work system collects the images of garage/yards and images of the entrance road to the garage/yard through the yard registration approval process using the "Pay as you park" mobile application. In this scenario, technically, the system must identify the parking yard surface type (asphalt, paved and unpaved) and the quality of each surface type (Standard, Average, Bad) to measure the standard of the parking yard surface (e.g., Asphalt Standard, paved average, etc). A special model was trained for the surface type classification with the following classes: asphalt, paved, and unpaved. The testing and model training was carried out on a computer using an NVIDIA GeForce GTX 960 graphics running on a Windows 10 operating system with 8 GB RAM. Convolutional Neural Networks $(\mathrm{CNN})$ is used to do the parking surface classification task in this method.

Three distinct models were utilized for surface quality for each type of surface. The structure of all four models is the same. The output of the first model is referred to as the particular quality model. A Region of Interest (ROI) (Figure 5 ) is defined in the pre-processing step for each input image before the CNN structure because it does not need the entire image to categorize the parking surface.

The goal of this ROI is to save just the parts of the image that include parking surface pixels. Because in certain frames there are included unnecessary objects that affect the training model badly. So, the top half of the image, as well as a small part of the image bottom have been neglected because of this reason.

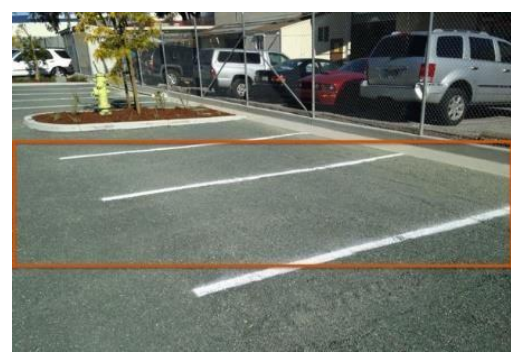

Figure 5 - Region-of-Interest

As Shown After that, each frame's brightness is increased or decreased as part of the data augmentation. This improves the training input set and aids the system in learning to recognize the same kind and parking surface types under various lighting situations. After all, the input surface images are passed through a CNN structure that consists of three convolution layers and two fully linked layers. Then all images were transmitted to the training phase with information on width, height, and the number of channels in the first convolution layer. In all of the convolution layers, max-pooling is used to decrease the input dimensionality, which helps in the analysis of feature information in the inputs. After that, ReLU is used as an activation function at the end of each convolution layer. As the next step, the flatten layer converts the convolution multi-dimensional tensor to the one-dimensional tensor after the convolutional layers. Finally, two fully connected layers are added. A ReLU activation function is used in the first fully connected layer and the second fully connected layer contains the potential outputs, or desired classes, which will be three classes for parking surface type models, asphalt quality models, and paved quality models, and parking surface unpaved quality models. The probability of each class was calculated using the SoftMax algorithm because it makes greater sense in instances when subjectivity is absent, such as pavement type determination. In the end, the Adam optimizer is used to adjust the network weights based on the input frames used during the training process.

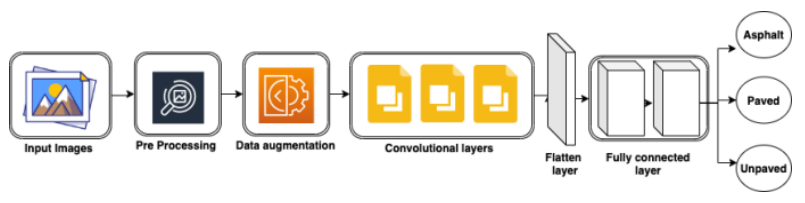

Figure 6 - The CNN Structure

\section{RESULT AND DISCUSSION}

\subsection{Tracking available parking spaces}

There were few algorithms to be considered in the process of identifying the availability of slots inside a parking area such as CNN, RCNN, Fast RCNN, Faster RCNN, and Mask RCNN. In the 'Pay as You Park' smart parking solution, Mask RCNN has been used due to its pre-trained ability and high accuracy rates in object identifying. The accuracy of the output is not high due to the lack of data set to train the model. But with a proper dataset, the model can get higher accuracy in the future. Figure 7 shows a sample identification of free parking slots of a parking area.

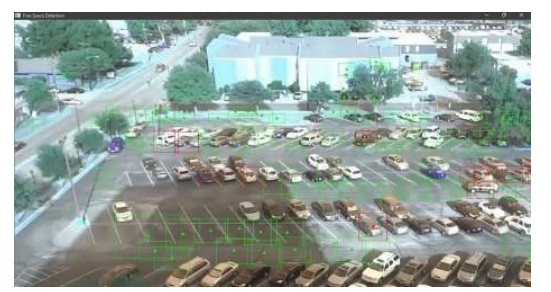

Figure 7 - Output (Available car space identification)

\subsection{Optimal Parking suggestions}

To achieve the objective of optimal parking yard suggestion, the authors identified the facts that need to be considered when suggesting a parking yard to the user. Distance, availability, and physical characteristic of the vehicle. To identify the nearest parking yard for a particular user Haversine is the best algorithm when considering both accuracy and computational cost. And by validating the vehicle's physical characteristics with parking slot and parking yard metrics this research suggestion will make sure that the vehicle could enter the parking yard and park. In order to identify the occupancy or availability of parking yards at a given time, SARIMA is used since it results in Accuracy with $97.7 \%$ and 0.019 validation loss. 


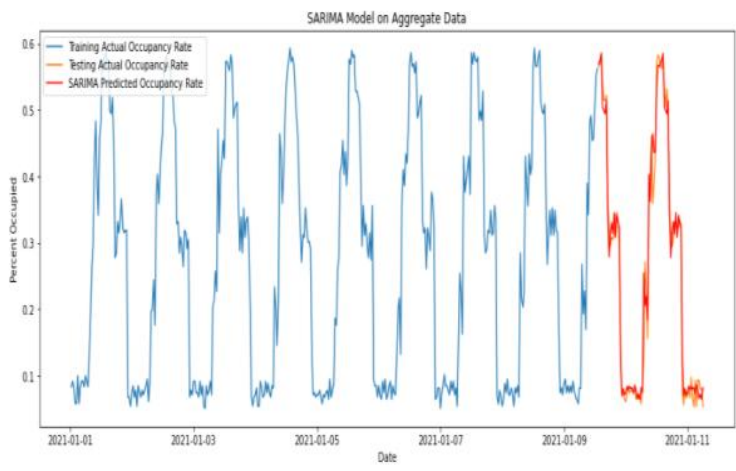

Figure 8 - SARIMA forecasting Graph

With the combination of all these facts considered, authors can suggest to the users the optimal parking yards to park their vehicle. It would impact the society and environment positively.

\subsection{Getting User's Position}

When considering getting the user's positions Authors have been used many algorithms such as DNN, NN, and XGBoost algorithms. Authors have used NN to train the model due to its high accuracy. DNN and XGBoost algorithms are low inaccuracy when getting the actual distance of a user. By using about 300 frames from a dataset, authors have been trained the models and the trained model accuracy of getting $\mathrm{X}$ value of a user is $100 \%$ and the accuracy of getting $\mathrm{Y}$ value of a user is $90 \%$.

\section{Table 2 - Accuracy of NN Model}

\begin{tabular}{|l|c|c|}
\hline Model Name & $\begin{array}{l}\text { Training } \\
\text { Accuracy of X }\end{array}$ & $\begin{array}{l}\text { Training Accuracy } \\
\text { of Y }\end{array}$ \\
\hline $\begin{array}{l}\text { Neural } \\
\text { Network } \\
\text { model }\end{array}$ & $100 \%$ & $90 \%$ \\
\hline
\end{tabular}

\subsection{Parking Yard Surface Validation}

\subsubsection{Parking Surface Classification}

For the training purposes, more than $6000+$ frames were used from the dataset to train the model for parking surface classification, which were manually divided into three categories as asphalt, paved, and unpaved. All training data were divided into two parts such as $80 \%$ for training and $20 \%$ for validation. The number of frames chosen for each class was proportionate to the amount of data available. Around $70 \%$ of the frames are asphalt, $20 \%$ are paved, and $10 \%$ are unpaved.

\section{Table 3 - Accuracy of Surface Type Model}

\begin{tabular}{|c|c|c|c|}
\hline $\begin{array}{c}\text { Model } \\
\text { Name }\end{array}$ & $\begin{array}{c}\text { Training } \\
\text { Accuracy }\end{array}$ & $\begin{array}{c}\text { Validation } \\
\text { Accuracy }\end{array}$ & $\begin{array}{c}\text { Validation } \\
\text { Loss }\end{array}$ \\
\hline $\begin{array}{c}\text { Surface Type } \\
\text { Model }\end{array}$ & $100 \%$ & $93.8 \%$ & 0.146 \\
\hline
\end{tabular}

\subsubsection{Parking Surface and Quality Classification}

When considering the surface quality, this method used three models for the surface quality classification which is used to identify the quality of each surface type. In the training stage, authors have used a few frames: $1500+$ frames for the asphalt quality model, 600+ frames for the paved quality model, and $500+$ frames for the unpaved quality model. Authors separated 80 percent of the data for training, and the remaining $20 \%$ was picked at random for validation.

Table 4 - Parking Surface Validation Model Accuracy

\begin{tabular}{|c|c|c|c|}
\hline Model Name & $\begin{array}{c}\text { Training } \\
\text { Accuracy }\end{array}$ & $\begin{array}{c}\text { Validation } \\
\text { Accuracy }\end{array}$ & $\begin{array}{c}\text { Validation } \\
\text { Loss }\end{array}$ \\
\hline $\begin{array}{c}\text { Asphalt quality } \\
\text { model }\end{array}$ & $100 \%$ & $88.4 \%$ & 0.011 \\
\hline $\begin{array}{c}\text { Paved quality } \\
\text { model }\end{array}$ & $100 \%$ & $96.9 \%$ & 0.190 \\
\hline $\begin{array}{c}\text { Unpaved quality } \\
\text { model }\end{array}$ & $100 \%$ & $92.7 \%$ & 0.206 \\
\hline
\end{tabular}

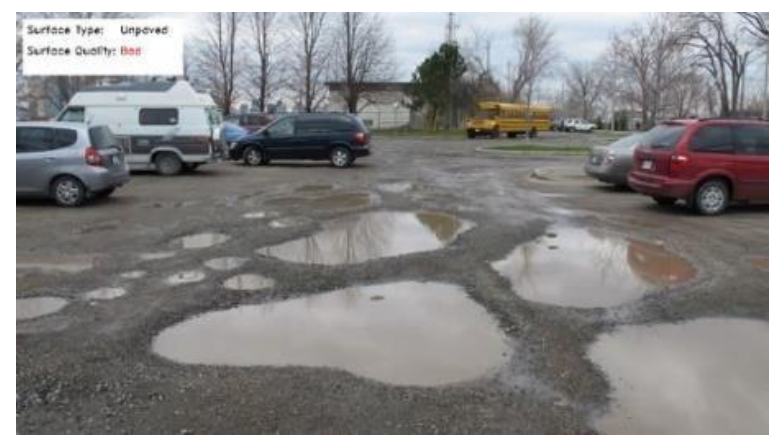

Figure 9 - Unpaved (Bad)

\section{CONCLUSION AND FUTURE WORKS}

As soon as the product is for placement in a live environment and since this is a considerable problem in Sri Lanka parking systems the users can get an advantage after the user pays the fee. According to the current parking systems in Sri Lanka, User has parking time of thirty minutes by thirty minutes payment packages or hour by hour payment packages. Consider if a user parked a vehicle in a parking area for ten minutes user will be charged for whole thirty minutes. As a unique feature in this proposed system, the user only must do the payment for the exact parking time. As future work, internal parking of two or more stories will be done.

\section{REFERENCES}

[1] Bhavani, D. S., \& Ghalib, M. R. "Internet of Things Based Smart Car Parking System Using K-Nearest Neighbour Algorithm to Find the Nearest Slot. Journal of Computational and Theoretical Nanoscience,"2018, 15(6), 2040-2045. doi:10.1166/jctn.2018.7403

[2] Gunasekara, G. G. Y. U., Gunasekara, A. D. A. I., \& Kathriarachchi, R. P. S. (2015). A Smart Vehicle Parking Management Solution.

[3] Karunarathne, M. S., \& Nanayakkara, L. D. J. F. (2014). A Prototype to Identify Availability of a Car in a Smart Car Park with Aid of Programmable Chip and Infrared Sensors. Journal of Emerging Trends in Computing and Information Sciences, 5(2).

[4] Nwave, (2021), Advantages and Disadvantages of Smart Parking Sensors | Nwave [Online] Available: https://www.nwave.io/news/pros- and-cons-of-smartparking-systems/ [Accessed $20 \mathrm{Feb} 2021]$

[5] Paidi, V., Fleyeh, H., Hakkansson, J., \& Nyberg, R. G. (2018). Smart parking sensors, technologies and applications for open parking lots: a review. IET 
Intelligent Transport Systems, 12(8), 735-741.

[6] M. A. P. Chamikara, Y. P. R. D. Yapa, S. R. Kodituwakku and J. Gunathilake, "An Efficient Algorithm to Detect The Nearest Location Of A Map For A Given Theme," 2013 International Journal of Scientific \& Technology Research.

[7] Y. Dian Harja and R. Sarno, "Determine the best option for nearest medical services using Google maps API, Haversine and TOPSIS algorithm," 2018 International Conference on Information and Communications Technology (ICOIACT), Yogyakarta, Indonesia, 2018, pp. 814-819, doi: 10.1109/ICOIACT.2018.8350709.

[8] Siahaan, Andysah P. U. 2017. "Haversine Method in Looking for the Nearest Masjid." INA-Rxiv. September 22.doi:10.31227/osf.io/eb3ja

[9] M. A. Kuhail, M. Boorlu, N. Padarthi and C. Rottinghaus, "Parking Availability Forecasting Model," 2019 IEEE International Smart Cities Conference (ISC2), 2019, pp. 619-625, doi: 10.1109/ISC246665.2019.9071688.

[10] Improve Indoor Positioning Accuracy Using Filtered RSSI and Beacon Weight Approach in iBeacon Network Laial Alsmadi Faculty of Engineering and IT University of Technology Sydney Sydney, Australia Xiaoying Kong Faculty of Engineering and IT University of Technology Sydney Sydney, Australia Kumbesan Sandrasegaran Faculty of Engineering and IT University of Technology Sydney Sydney, Australi

[11] Ibrahim, Hossam El-Din, Car Parking Problem in Urban Areas, Causes and Solutions (November 25, 2017). 1st International Conference on Towards a Better Quality of Life, 2017, Available at SSRN: https://ssrn.com/abstract=3163473or http://dx.doi.org/10.2139/ssrn.3163473
[12] M. M. Forrest, Z. Chen, S. Hassan, I. O. Raymond and K. Alinani, "Cost Effective Surface Disruption Detection System for Paved and Unpaved Roads," in IEEE Access, vol. 6, pp. 48634-48644, 2018, doi: 10.1109/ACCESS.2018.2867207.

[13] Nienaber, S \& Booysen, M.J. (Thinus) \& Kroon, Rs. (2015). Detecting Potholes Using Simple Image Processing Techniques and Real-World Footage. 10.13140/RG.2.1.3121.8408.

[14] Taluja, Chandan \& Thakur, Ritula. (2018). An Intelligent Model for Indian Soil Classification using various Machine Learning Techniques. 2250-3005.

[15] Lee, T.; Yoon, Y.; Chun, C.; Ryu, S. CNN-Based RoadSurface Crack Detection Model That Responds to Brightness Changes. Electronics 2021, 10, 1402. https://doi.org/10.3390/electronics10121402

[16] Varona, B., Monteserin, A., \& Teyseyre, A. (2019). A deep learning approach to automatic road surface monitoring and pothole detection. Personal and Ubiquitous Computing. doi:10.1007/s00779-019-01234Z

[17] viso.ai, (2021), Mask R-CNN: A Beginner's Guide | viso.ai [online] Available: https://viso.ai/deeplearning/mask-r-cnn/ [Accessed 29 August 2021]

[18] Smart Parking System Based on Bluetooth Low Energy Beacons with Particle Filtering Andrew Mackey, Student Member, IEEE, Petros Spachos, Senior Member, IEEE, and Konstantinos N. Plataniotis, Fellow, IEEE

[19] Bluetooth-based Indoor Navigation Mobile System Adam Satan Institute of Information Science University of Miskolc Miskolc, Hungary satan@iit.uni miskolc.hu. Satan, A. (2018). Bluetooth-based indoor navigation mobile system. 2018 19th International Carpathian Control Conference (ICCC). 